\title{
Clear aligners in orthodontics
}

\author{
G. Dharmadeep ${ }^{1}$, M. Balagangadhar ${ }^{2, *}$, C. Madhuri $^{3}$, Y. Muralidhar Reddy ${ }^{4}$, G. Kranthi Praveen Raj $^{5}$ \\ ${ }^{\mathbf{1}}$ Assistant Professor, Dept. of Orthodontics, G. Pulla Reddy Dental College, Andhra Pradesh, ${ }^{2}$ Assistant Professor, Dept. of \\ Orthodontics, ${ }^{2}$ Yogita Dental College and Hospital, Maharashtra, ${ }^{3}$ Assistant Professor, Dept. of Orthodontics, Geetam Dental \\ College and Hospital, Visakhapatnam, Andhra Pradesh, ${ }^{4}$ Professor and Head, ${ }^{5}$ Associate Professor, Dept. of Orthodontics, G. \\ Pulla Reddy Dental College, Andhra Pradesh, India
}

*Corresponding Author:

Email: baalagangadhar@gmail.com

\begin{abstract}
When we hear the word "orthodontics," what comes to mind is probably a young teenager whose teeth are covered by a latticework of metal. However, there now exists an increasingly popular alternative to traditional metal braces: Clear bite aligners. This article describes the clearbite aligner technique, the technology behind clearbite aligners, what it is, how it works, process, records which are essential for case submission, case selection and advantages of clearbite Aligners are highlighted. The clearbite aligner clinical technique, procedure, essential tips are described and cases treated with clearbite Aligners are presented.
\end{abstract}

Keywords: Aligners, Invisible orthodontics, Clear aligners, Clearbite.

\section{Introduction}

When we hear about "orthodontics," what comes to mind is... a latticework of metal covered on the teeth. However, because of increased aesthetic concern to get orthodontic treatment done in an invisible way, clear alternative to those traditional metal braces has arised: Clear aligners

\section{What are clear aligners $?^{1-3}$}

Clear aligners are also one of the orthodontic appliances to straighten teeth, but these are invisible alternative of traditional metallic braces where patients wear a series of removable medical grade clear plastic trays made of CAD CAM Technology.

How do clear aligners work ? $^{5-8}$

Clear aligners also work as the same way traditional orthodontic appliances work by applying carefully controlled forces to teeth which are determined by software to move them into a better position. Instead of metal, they are made of clear, flexible plastic.

Here's how clear aligners work: By using CAD CAM Technology each case the best possible alignment will be done. According to the complexity of the case and the movements which are given for correction of malocclusion, number of stages will be decided. For each stage, invisible plastic mouth "trays" or "aligners," will be fabricated. The patient should wear these aligners for at least two weeks, for a minimum of 21 hours per day and then move on to the next set of aligners in series to accomplish the next stage of gradual movement until all the teeth are correctly aligned.

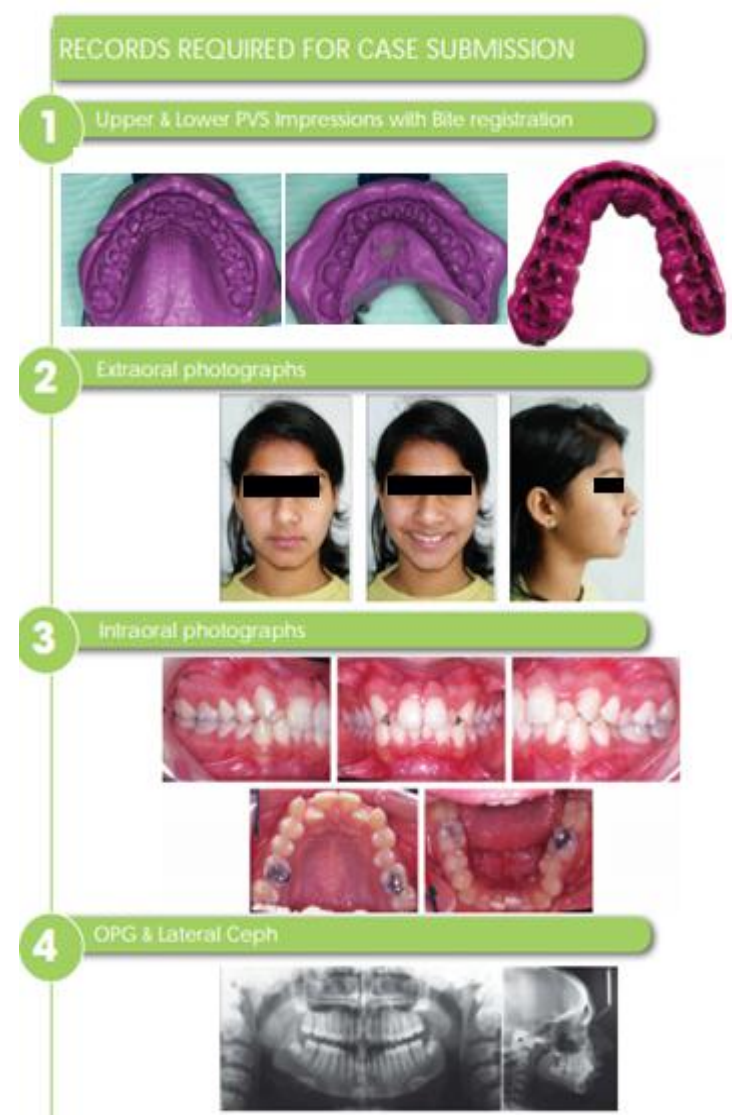

Fig. 1:

\author{
Advantages of clear aligners \\ Invisible \\ Treatment at any age \\ Comfortable \\ Flawless smile \\ No food restrictions \\ Professional friendly \\ Process of clear aligners
}


History and Clinical Examination

Patient Selection

Treatment Records Submission

Treatment plan Confirmation

Virtual Setup

Aligner fabrication and delivery

Treatment monitoring

Retention

\section{Case Selection}

A thorough knowledge on biomechanics of orthodontic tooth movement with aligners is very important to achieve successful treatment planning with regard to staging and setups.

A common misconception is that the manufacturer of the aligners is what provides a good smile result. Rather, it is the orthodontist who works on the treatment through thorough diagnosis and treatment planning, staging clinical observation, and follow up with managing the tracking issues that provides a good result. In other words, all clear aligners can be effective in the hands of an experienced clinician.

Another misconception is that the orthodontist merely needs to dispense the aligners the manufacturer provides, however, treatments in which all of the aligners are made in advance rarely work out as planned showed to you in the virtual plan. Because biology of humans is complex and not as predictable as computer models. For this reason, the treating clinician needs to be involved throughout treatment in order to provide a correct diagnosis, treatment staging, and setup, as well as the necessary refinements and followup appointments to make sure the treatment is going as planned.

\section{Before Starting Clear Aligners Treatment Comprehensively Inform the Patients About}

1. Self-Motivation. Because it is a removable appliance, the treatment outcome will be depending on how much the patient is motivated towards the appliance.

2. Attachments. Discuss attachments with patients at the consultation. Inform patients about what they are, and where and when they will be placed. Attachments are small pieces of light-cured composite resins bonded temporarily on the tooth surface which helps in tooth movement and aligner retention. Initially, the technician designs a virtual resin which is transformed into a stereolithographic model and an aligner called the template aligner is fabricated from the same, this is used to make the actual attachments in the patient's mouth.

3. Interproximal reduction (IPR). Educate patients about different space gaining methods and inform about what IPR is, and why it is necessary in their treatment. IPR is not only for space gaining but used to remove tight contacts

\section{During Routine Appointments Procedure}

1. Carefully monitor the progress of treatment. Not all patients will follow the suggested progression of aligners because biology and physiology of patients are various and, at times, unpredictable.

2. Advance carefully to the next aligner. Aligners must fit loosely before advancing to the next one. Advancing too rapidly is a common cause of tracking issues. If any tracking issue is detected should be corrected before advancing to the next set of aligners. If discrepancy is less $(1 \mathrm{~mm})$ should be managed by making patient to wear for some more days. If discrepancy is more (greater than $1 \mathrm{~mm}$ or if the issue doesn't corrected even after using for more days) detail pliers or auxiliary techniques should be used to correct the condition.

\section{Essential Tips to be Informed}

1. Patient should wear aligners for at least $20-22$ hours in a day except when the patient is eating, drinking, flossing to achieve desired outcome.

2. Should take proper care of clear aligners. If any aligner lost or broken should not proceed to next aligner. Till the lost aligner comes from lab need to wear previous aligner.

3. To prevent plaque or food remains to stick between teeth and aligners should have a tooth brush handy for proper cleaning of teeth every time after eating or drinking.

4. While wearing aligners, except water should not take any other beverages such as tea, coffee, cola or wine as they will stain the aligners.

5. For cleaning aligners don't use hot water because it can disfigure their shape and loose its properties. Avoid tooth paste for cleaning, instead, brush them with a moderate detergent or anti-bacterial wash.

6. While taking out aligners should be careful. Do not take them out forcefully. Use light hands at inner surface and remove gently.

7. It is very important to wear retainers after treatment to restrict newly positioned teeth from getting back to their original position. 


\section{Cases treated with clear bite aligners}
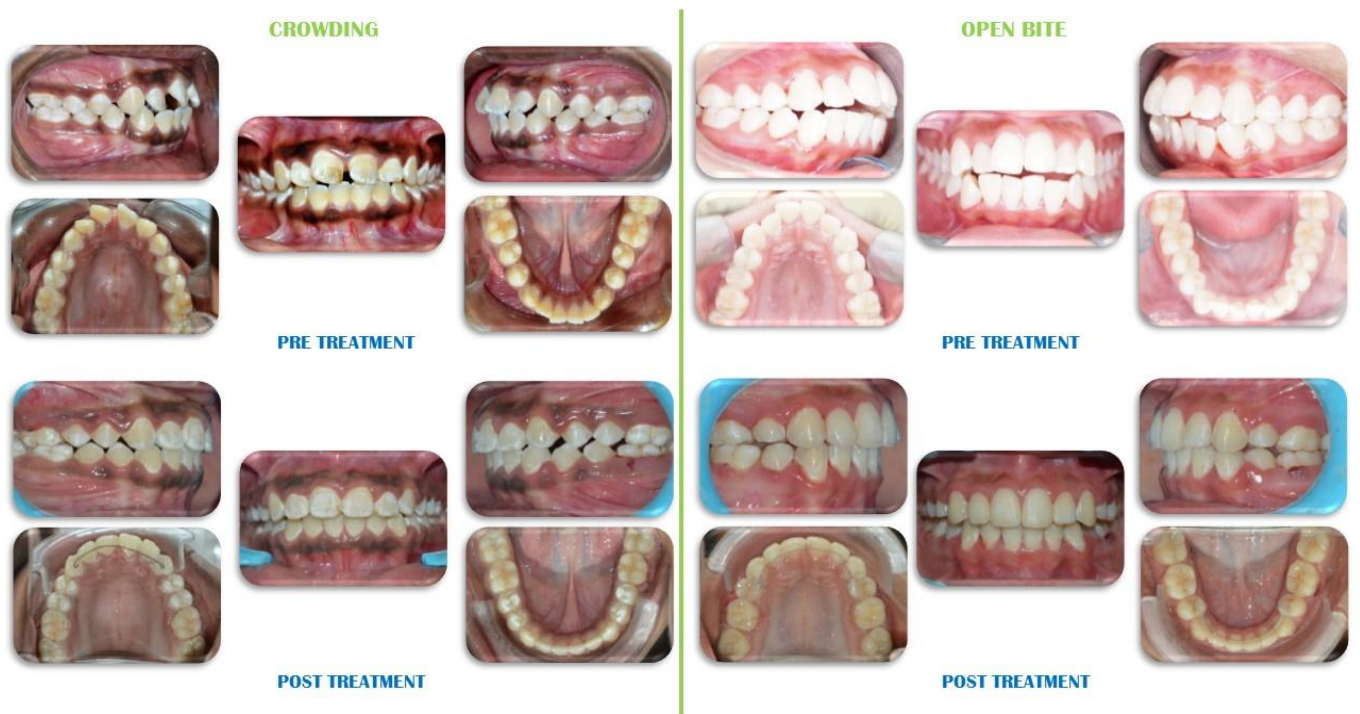

POST TREATMENT

POST TREATMENT
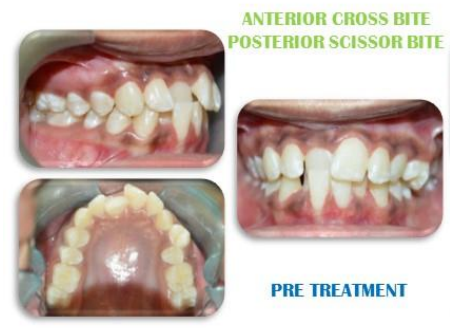

PRE TREATMENT
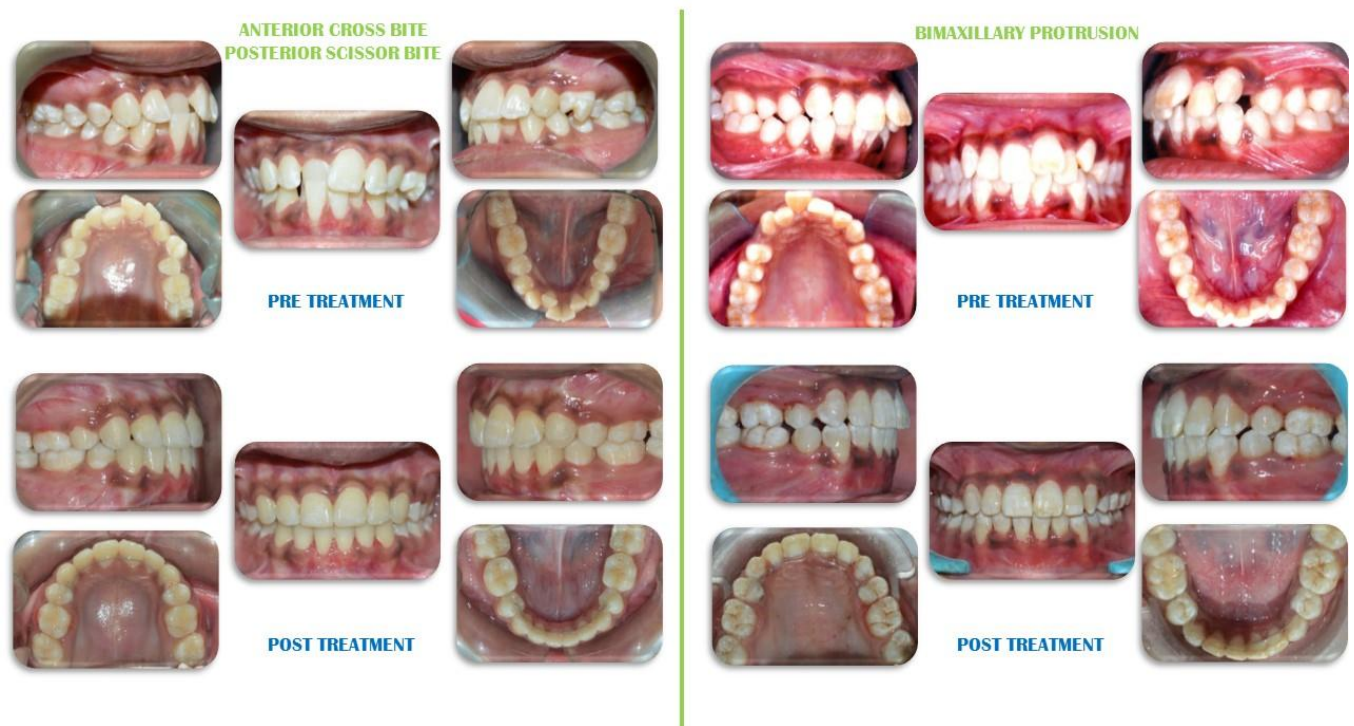

Fig. 2:

\section{Conclusion}

Aligners are also one of orthodontic appliances which need Orthodontist diagnosis and treatment planning, monitoring and corrections of tracking issues at regular appointments. So that only the results can be achieved properly.

5. Rinchuse DJ, Rinchuse DJ. Active tooth movement with Essix-based appliances. J Clin Orthod 1997;31(2):109-12.

6. Sheridan JJ, Ledoux W, McMinn R. Essix technology for the fabrication of temporary anterior bridges. J Clin Orthod 1994;28(8):482-6.

7. Ballard R, Sheridan JJ. Air-rotor stripping with the Essix anterior anchor. J Clin Orthod 1996;30(7):371-3.

8. Rinchuse DJ, Rinchuse DJ. Active tooth movement with Essix-based appliances. J Clin Orthod 1997;31(2):109-12.

\section{References}

1. Kesling HD. The philosophy of the tooth positioning appliance. Am J Orthod 1945;31:297-304.

2. Ponitz RJ. Invisible retainers. Am J Orthod 1971;59(3): 26672.

3. McNamara JA, Jr, Kramer KL, Juenker JP. Invisible retainers. J Clin Orthod 1985;19(8):570-8.

4. Sheridan JJ, LeDoux W, McMinn R. Essix retainers: fabrication and supervision for permanent retention. J Clin Orthod 1993;27(1):37-45. 